\title{
Code is Law: Subversion and Collective Knowledge in the Ethos of Video Game Speedrunning
}

\section{Michael Hemmingsen}

To cite this article: Michael Hemmingsen (2020): Code is Law: Subversion and Collective Knowledge in the Ethos of Video Game Speedrunning, Sport, Ethics and Philosophy, DOI: 10.1080/17511321.2020.1796773

To link to this article: https://doi.org/10.1080/17511321.2020.1796773

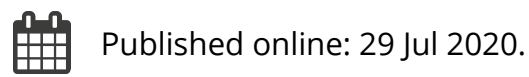

Submit your article to this journal $\square$

Q View related articles ๘

View Crossmark data $₫$ 


\title{
Code is Law: Subversion and Collective Knowledge in the Ethos of Video Game Speedrunning
}

\author{
Michael Hemmingsen (iD \\ College of Liberal Arts \& Social Sciences, University of Guam, Mangilao, GU, USA
}

\begin{abstract}
Speedrunning is a kind of 'metagame' involving video games. Though it does not yet have the kind of profile of multiplayer esports, speedrunning is fast approaching e-sports in popularity. Aside from audience numbers, however, from the perspective of the philosophy of sport and games, speedrunning is particularly interesting. To the casual player or viewer, speedrunning appears to be a highly irreverent, even pointless, way of playing games, particularly due to the incorporation of "glitches". For many outside the speedrunning community, the use of glitches appears to be cheating. For speedrunners, however, glitches are entirely within the bounds of acceptability. Because of this, however, speedrunning frequently involves sidestepping what are typically taken to be the core challenges of the game. By examining the distinction between the use of glitches and cheating in speedrunning, we can gain a greater understanding of the unique ethos of this activity; that is, we can make sense of what fundamentally constitutes speedrunning as a metagame. I argue that by understanding the code of the game not as rules but as physics, and by examining what actions are deemed impermissible by the speedrunning community - such as hardware modification and hacking - we can see that the ethos of speedrunning has three components: constitutive skills (including dexterity, memorisation and mental fortitude); a collective, finegrained knowledge of the game and the desire to subvert the intentions of the programmers. Each of these components limits and structures the earlier ones: collective knowledge takes priority over constitutive skills, and subversion takes priority over both. These three components form the ethos that structures speedrunning as a metagame, expressing what speedrunners take to be its central aim.
\end{abstract}

\section{KEYWORDS}

Video games; speedrunning; subversion; collective knowledge; metagame

\section{Introduction}

Speedrunning is a kind of 'metagame' involving video games. By 'metagame' I mean a game that takes an existing game and reconfigures it by adding player-designed rules and objectives. In the case of speedrunning, the player-defined goal is that the game should be 'completed' (and what this means depends on the specific speedrunning community and category of play) in the shortest possible time. 


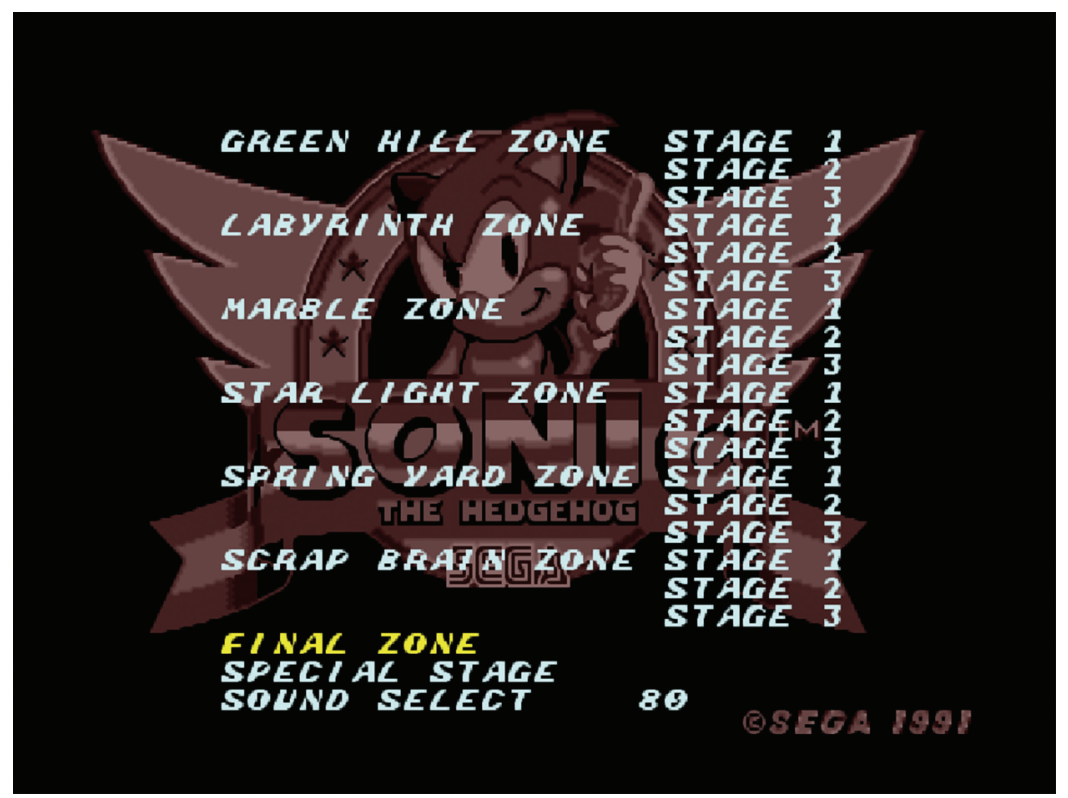

Figure 1. Sonic the Hedgehog Level Select Screen.

The status of speedrunning as a 'metagame' is particularly important to note in this paper. As I discuss in more detail later, speedrunning is not, in a significant sense, 'playing the game'. While it would be too strong to say that the specific software is irrelevant in speedrunning, nonetheless it is important to note that the game is being used as a toolor perhaps as an 'environment' within which speedrunners are playing a metagame of their own creation. In this sense, then, it is necessary to separate the metagame of speedrunning from the game as software. In fact, though we use the term 'game' to refer to the virtual environment in which speedrunning is taking place, this sense of 'game' should not be considered the same as that used in the philosophy of sport. In other words, the software itself should not be considered a 'game' for the purposes of this discussion in the sense that rugby, cricket, chess, or speedrunning are games.

With the exception of showcase events such as Games Done Quick, speedrunners do not generally compete directly. Rather, they compete asynchronously, attempting to achieve the fastest times in their game and category. These scores are tracked on online leaderboards, such as at speedruns.com (Snyder 2017, 9).

Currently most competitive speedrunning involves 'Real Time Attack' (RTA) runs. In RTA, players use external timers to measure the length of their runs, with agreed upon points for when the timer should be activated and deactivated. This equipment can be quite sophisticated, recording scores to the millisecond (as in running, for instance, world records in many games are beaten by fractions of a second).

The objectives of the game are decided on by the speedrunning community. Typically, games are divided into multiple categories with differing objectives, most commonly 'Any $\%$ ' and ' $100 \%$ '. 'Any\%' involves triggering the final cutscene or game credits by the shortest means. By contrast, '100\%' categories typically require runners to collect 'all optional upgrades and items before completing the game' (Snyder 2017, 15). While 
these are the limit cases for categories, communities can and do create categories requiring objectives of various different kinds, though typically only when a new category would significantly change the speedrun by requiring very different 'routes' through the game or emphasising different skills. Additionally, categories are typically separated into 'glitched' and 'glitchless', a distinction that I will discuss further later in the paper.

Speedrunning is an increasingly popular kind of metagaming. Though it does not yet have the kind of profile of multiplayer e-sports-multiplayer games like Overwatch (2016) and Starcraft II: Wings of Liberty (2010), for instance, have large, well-funded tournaments -speedrunning is fast approaching e-sports in popularity. For example, the live speedrunning showcase, Games Done Quick, reported an attendance of 2,200 for their 2019 event, with a peak viewership, via the streaming platform Twitch, of 219,240 (Good 2019). This rose to 237,523 for their 2020 event (GDQStat 2020). By comparison, the Overwatch League (one of the most popular e-sports leagues) had a viewership of 313,000 for their 2019 final (Illingson 2019). Direct comparison between the two numbers can be difficult. Nevertheless, speedrunning has a significant and, importantly, growing audience. Speedrunning is also now a semi-professionalised activity, with some full-time speedrunners able to make a living from the activity. It is timely, then, for philosophers to seriously engage with the activity.

Aside from audience numbers, however, from the perspective of the philosophy of sport and games speedrunning is particularly interesting. To the casual player or viewer, speedrunning appears to be a highly irreverent, even pointless, way of playing games. For some, it seems as if speedrunners are not 'truly' playing the game due to the incorporation of 'glitches' into speedrunning. A glitch can be thought of as a 'flaw in the programming of a game exploited for the benefit of the runner' (Snyder 2017, 23-4). This may include, among other things, 'clipping' glitches, where the player-controlled avatar is able to move through walls, floors or doors that are intended to bar their way; or 'wrong warps', where the player manipulates the game into send a player to an unintended area, often skipping large portions of the game in the process.

For many outside the speedrunning community, the use of glitches appears to be cheating. That is, it seems as if the player is breaking the game is some way. For speedrunners, however, glitches are entirely within the bounds of acceptability. In fact, glitches are a central feature of speedrunning; for most games, a glitchless performance of a game would have no hope of beating the world record time. Nevertheless, speedrunning frequently involves sidestepping what are typically taken to be the core challenges of the game. Certainly, speedrunning undermines any sense of continuity or plot. For instance, in role-playing games (RPGs), where narrative is often taken to be the central pleasure of the game, speedrunners will play the game in Japanese or Chinese (even if they do not read or speak these languages), because Japanese and Chinese have fewer characters, meaning that textboxes are shorter and scroll more quickly, savings the runner precious seconds over the course of a run (Snyder 2017, 31).

From a philosophy of sport and games perspective, the incorporation of glitches is provocative. By examining the distinction between the use of glitches and cheating in speedrunning, we can gain a greater understanding of the unique ethos of this activity: that is, the 'set of unofficial, implicit conventions which determine how the rules of a game are to be applied in concrete circumstances' (D'Agostino 1981, 15); or the 'socially determined interpretation' (Kreider 2011, 55) of the purpose of the activity that regulates 
how we ought to apply the rules in practice and deal with novel scenarios. By identifying the ethos of speedrunning, we can make sense of what fundamentally constitutes speedrunning as an activity.

Importantly, 'ethos' is not the same as 'rules'. Speedrunning-like any game-has both an ethos and rules. By 'ethos', then, I mean the basic values of the activity-the 'spirit of the game' - that explains, generates and justifies the rules that the gamewrights (here, the speedrunning community) create. So, for instance, 'glitches are permitted' is a rule, but this rule is generated and made sense of by a set of values. While I am interested in the rules of speedrunning in this paper, I am only interested in them insofar as they shed light on the ethos of the activity.

Because of the acceptance of glitches in the metagame, speedrunning can initially be taken to be an activity in which anything that can be done is permissible. However, I argue that this is not quite right. By pushing at this idea, though, we can see what makes speedrunning such an interesting and important subject of analysis. How should we understand glitches? If glitches are permissible, how is cheating understood in speedrunning, and why? I argue that by understanding the code of the game not as rules but as physics, and by examining what actions are and are not permitted by the speedrunning community, we can see that the ethos of speedrunning has three components: constitutive skills (including dexterity, memorisation and mental fortitude); a collective, finegrained knowledge of the game mechanics and code; and the desire to subvert the intentions of the programmers. Each of these components limits and structures the earlier ones: collective knowledge takes priority over constitutive skills, and subversion takes priority over both. These three components form the ethos that structures speedrunning as a metagame, expressing what speedrunners take to be its central aim and spirit.

The latter two components of speedrunning-collective knowledge and an explicit aim to subvert the intentions of the programmer of the game-are, if not unique to speedrunning, at least unusually prominent. For this reason, I suggest that speedrunning should be of particular interest to philosophers of sport.

\section{Ethos or Ethoi?}

One question that might be raised at this point, however, is whether it is meaningful to speak about 'an' ethos of speedrunning. After all, speedrunning involves hundreds of different games; at the time of writing, speedrun.com hosts runs of over two thousand games with active players (though most games have only one or two). Furthermore, each game will typically have multiple categories, including both glitched and glitchless variants. Given this, perhaps it makes little sense to think of speedrunning as having a single ethos. Perhaps different games and categories are quite distinct activities with unique aims and rationales.

I think this is mistaken, however, for two reasons. First, while different games and categories may involve a different balance of constitutive skills, the basic skills of speedrunning are consistent across all. Furthermore, the three components I identify-constitutive skills, collective knowledge and subversion-have the same relationship to each other, regardless of the game or category.

Secondly, every game contains glitched categories and in many ways the glitched categories are viewed as the 'standard' or 'default' category. New glitchless categories 
may be created in order to preserve certain modes of play when significant glitches are discovered, but the glitched category is still acknowledged as the fastest category. Since speed is the goal of a run (just as speed is the goal of a swimming race, for instance), then the fact that glitchless categories are faster than glitched categories matters. In some ways, we can think of glitchless categories as the equivalent to the 'freestyle' category of swimming: even if the accomplishments of top butterfly swimmers are extremely impressive, nothing changes the fact that freestyle represents the height of accomplishment in swimming in general. Similarly, holding a world record in a glitchless category is impressive, but a glitched world record in that game represents the best expression of speedrunning that game in general. Most of my focus here is therefore on glitched speedrunning. Nevertheless, much of what I will say can be applied, suitably modified, to glitchless speedrunning as well.

\section{Code is Law}

Understanding the ethos of speedrunning is especially interesting and challenging since actions that seem to the casual gamer as outside the bounds of permissibility are standardly performed in speedruns. Any glitch in the code-from walking through supposedly impassable walls to skipping almost all the game by exploiting unintended bugs in the programming-are acceptable (Brewer 2017, 52). For casual gamers, such actions go so far outside the game as intended by the programmers that it is difficult to understand what the purpose is of playing in such a way, especially when the glitches skip over or reduce the central challenges of the game.

The question is, then: what does the inclusion of glitches in speedrunning (and the exclusion of other actions outlined in the next section) tell us about the fundamental nature of speedrunning as a metagame?

Perhaps the central idea in speedrunning is contained in the name: it is about speed. Whatever allows the player to go from the starting point to the designated endpoint (while achieving whatever other objectives are required by the particular category of play), is permissible. In this sense, it can be said that 'code is law' for (glitched) speedrunning. That is, if the code allows it, then it is permissible. ${ }^{1}$

However, the phrase 'code is law' can be taken in different ways. We can think of law in the sense of 'rule', and we can think of law in the sense of 'law of nature'. I suggest that code in speedrunning should be (mostly) understood in the latter sense: rather than being rules of the game, code is the physics under which the metagame operates. ${ }^{2}$

Thinking of code as physics can more easily make sense of the permissibility of glitches. For instance, when Seb Franklin suggests that speedrunning allows players to 'pass through walls that are coded to be impassable, harmlessly touch enemies that are coded to do a particular amount of damage [...] and [...] assuredly collect items that are coded to be random' (Franklin 2009, 176), Rainforest Scully-Blaker points out that this is not accurate. In his words, 'speedrunners do not pass through walls that are coded to be impassable ... a wall that is passible is coded as such' (Scully-Blaker 2016, 4). In other words, if the code did not allow an avatar to pass through a wall, they would simply not be able to do so. The fact that they can do so in some cases tells us that, in those cases, the code does allow the avatar to pass through the wall. This may not be what a casual player expects to happen, but the mere fact that the avatar can do it tells us that it is in the code. 
Of course, the programmers may have intended the wall to be impassable. But the fact that authors of a text-including game texts-intend others to interact with the text in a specific way does not constitute rules for doing so. For instance, authors intend readers to read the chapters of a book in a certain order. But we are perfectly free to abandon that order if we choose. If we do so, we will almost certainly gain a different meaning from the text than the author intended, for better or worse. Since speedrunning is a metagame that uses the code to play a different game of the speedrunners' own creation, there is no reason why the speedrunner should take the intentions of the programmer as binding on them. In fact, the question of whether glitches constitute cheating in the game according to the programmers is simply irrelevant to speedrunning. As a metagame, speedrunning is simply a different game to that which the programmers intended; it is a distinct thing.

The use of glitches in speedrunning can therefore be usefully compared to something like the Fosbury Flop in high jumping. Prior to the invention of the Fosbury Flop jumping technique by Dick Fosbury, it did not occur to high jumpers to jump in that way. But because the Fosbury Flop is superior to the previously used high jumping techniques, it is now the standard style. Unless and until a better technique comes along, high jumpers are required to use it if they want to be competitive. Despite being highly novel, then, the Fosbury Flop was perfectly allowable: Dick Fosbury simply took advantage of the laws of physics in a new and unexpected way.

We can think of glitches in speedrunning in much the same way. The ability of a player to pass their player through a seemingly impassable wall is not breaking any humanmade rules but is merely taking advantage of the 'laws of nature' of the game in a new and unexpected way. ${ }^{3}$ Speedrunners are not performing a forbidden action, since laws of nature have no role for 'permissible' and 'forbidden'; they are about what is possible or impossible. ${ }^{4}$

However, one key difference between real-world physics and 'code' physics-in appearance if not in principle-is that real-world physics is complete and comprehensive, whereas code physics is much looser and more undeveloped. What I mean by this is that in general in sports physics behaves in a fairly predictable way. There is little that one can do to bring about a drastically unexpected outcome. The flight of a ball, for instance, can be predicted to a very high degree of accuracy with knowledge about weight, wind and so on.

In game 'physics', however, the programmers are not in a position to design worlds in which actions will always have the consequences they intend. They create code that is good enough for the game, so long as the player stays within the intended parameters of that game; but that same code will almost certainly lead to strange consequences if the player does not. The framework of the code is messy-and will always be messy, no matter how much care the programmers take-and so the effects of pushing at the code end up much more extreme and unexpected than in the real world. For instance, it is unlikely that a rugby player will be able to discover a technique for walking through the opposing player as if they were not there; or that will transport them from one end of the field to the other in a single step. Yet, these are just the kinds of things that can be possible due to the looseness of game code.

Due to the extreme results of exploiting the game's code, it is therefore natural to compare game glitches with impermissible action in sport. For instance, James Newman 
compares Rocket-Jumping - a technique discovered originally in Quake (1996), where the player uses explosives to propel themselves at unusually high speeds or to previously unreachable heights - to performance-enhancing drugs; and claims that sequence breaking - the use of glitches to complete objectives out of their original intended order, or to skip parts of the game entirely-is akin to 'finding a shortcut and missing out half of the race' (Newman 2008, 138).

However, I think that these are mistaken comparisons. The desire to compare these unexpected outcomes to significant acts of cheating in sport comes from the fact that, again, our ability to exploit real-world physics is simply much more limited than our ability to exploit game code, due to the latter's relative simplicity and lack of comprehensiveness. For instance, the Fosbury Flop improves on the previous style of high jumping by applying physics in a novel way; however, the difference between the Fosbury Flop and the scissor technique are relatively minor in terms of their results. By contrast, the ability to glitch through a wall into a previously unreachable area is a much more obvious and significant difference from what is expected.

Ultimately, though, exploiting glitches in speedrunning is the same kind of thing as developing a new technique in a sport. It is more closely analogous to the modern forehand in tennis, or the invention of slam dunking in basketball, than it is to the use of performance enhancing drugs or taking an illegal shortcut (even when the glitch literally involves finding a shortcut, such as in Mario Kart 64 [1996]). As such, anything that does not 'change the code but rather exploit[s] the peculiarities of its construction and implementation' (Newman 2008, 136) is-and should be-permissible in speedrunning.

At this point, however, it might be wondered whether speedrunning still has a place for lusory means. As Suits (2005) argues, games_to be games at all-must involve achieving the pre-lusory goal using a means that is less than perfectly efficient. Otherwise, we are not playing a game, but simply engaging in everyday life. To use a well-known example, golf is not golf unless one hits the ball into the hole with the club; if we could simply walk to the green and place the ball in with our hands, would we even be playing a game at all (Pfleegor and Roesenberg 2014, 2017)? If anything is allowed in speedrunning, then perhaps it could be argued that we have abandoned the idea of lusory means.

This criticism, however, ignores the fact that the game is only one part of the activity of speedrunning. Just as important is the interaction between the player and the code. In this respect, then, if our pre-lusory goal is to get the player avatar to the ending scene of a game in the shortest possible time, the most efficient way of doing this is to simply hack the game in such a way as to load that ending scene immediately upon starting the game. Getting to that ending scene via inputting commands through a controller is a much less efficient means of achieving the same goal. In this way, then, while anything goes in terms of the code-since code is law-this does not mean that speedrunning does not involve lusory means or a lusory attitude. In fact, those who are found to cheat are excluded from the community precisely because they have failed to uphold the lusory attitude of speedrunning.

In short, to use the swimming analogy again, we can think of the code of the game as the basic physics underlying the activity, such as the viscosity of the water, the weight of gravity, and so on. ${ }^{5}$ Whether or not the category allows glitches-and to what extent-corresponds to the difference between freestyle and other stroke style categories. The aim of the run, i.e. 
'Any\%' vs. '100\%', parallels the distance swum, e.g. $100 \mathrm{~m}$ vs $10,000 \mathrm{~m}$ races. Finally, the kinds of considerations outlined in the next section-how one is allowed to interact with the game code-represents the rules in swimming that make the competition fair, e.g. the ropes that separate swimmers, rules about when one can enter the pool, against interacting with other swimmers, about the depth of the pool, the width of the lanes, and so on.

\section{Cheating in Speedrunning}

While 'anything goes' in terms of the manipulation of the game code, the player cannot simply do whatever they want. Certain player actions are impermissible and void the run. There are four main categories of impermissible action in speedrunning: hardware modification; code modification; run deception; and 'cheat' codes.

\section{Hardware Modification}

Hardware modification covers a range of activities. For instance, in RTA runs factors such as loading times, or 'lag'-slowdown in displaying the frames of the game due to the amount of information that needs to be rendered on the screen at any given time-can be affected by the hardware used, meaning that game run on a more powerful machine has the potential to have a shorter time than a game played on a less powerful one. For this reason, runs - to be competitive-typically must be done on the original system (usually a console) that the game was released on (though in-game techniques for reducing loading times and lag, such as altering camera angles so fewer objects are rendered on the screen, are permissible).

Another kind of hardware modification is anything that involves 'interacting with the game other than through the gameplay itself' (Speed Demos Archive 2019). For example, in The Legend of Zelda: Ocarina of Time (1998), players can sometimes gain an advantage through 'cartridge tilting', e.g. 'pulling up the side of your cartridge to make Link [the player avatar] glitch out (in a beneficial way)' (Snyder 2017, 163).

Also impermissible is 'RAM-watch', which is a method for monitoring RAM (random access memory) values in real-time. RAM-watch can be highly beneficial to a player, especially in manipulating RNG (random number generator) values, which are usually opaque to the player. This does not mean that the manipulation of RNG is forbidden in speedrunning, however, merely that it cannot be aided by external systems such as RAM-watch.

Players may also not use controllers that have features that are unavailable on the original system. For instance, some controllers have a 'turbo fire' feature that allows the player to 'press' a button rapidly by holding down the button with the turbo fire feature engaged. Others allow the use of 'scripts', i.e. permit a complex sequence of inputs to be recorded and mapped to a specific button (Brewer 2017, 43).

\section{Hacking}

The second category of cheating consists in modifications to the code. In short, altering the code of the game is not allowed (Brewer 2017, 34). So, for example, 
If a player were to use a technique ... that allowed them to clip through a wall, skipping portions of an area, then that would not be cheating. On the other hand, if a player were to clip through the same wall by accessing a command prompt and putting in a code that allowed them to do so, then that would be cheating (Brewer 2017, 36).

The basic idea is that the player should not be adding or modifying anything in the game code itself but should be interacting with the code as is.

\section{Run Deception}

The third category of impermissible action is run deception. Run deception involves deceiving the judges and viewers as to the veracity of the final run as presented. Perhaps the most common form of run deception is 'splicing', or 'the use of a program to piece together segments of a different run and pass it off as one "complete" speedrun' (Brewer 2017, 46).

\section{Cheat Codes}

The fourth category of impermissible action in speedrunning is the use of 'cheat codes'. Among other things, cheat codes include level/stage select codes, which allow players to choose which level to start the game at; and secret inputs that give the player's avatar additional abilities or items, such as allowing the avatar to pass through walls or makes them impervious to damage.

\section{The Ethos of Speedrunning}

Given the above, I suggest that there are three main components to the speedrunning ethos: in order of increasing importance, they are skill, collective knowledge and subversion. I will discuss each in turn.

\section{Skill}

There is no question that speedrunning involves several different skills, though these are found in varying proportions depending on the game being played. The most obvious skill is the dexterity required to control the avatar in an extremely precise way. The feats accomplished by the top speedrunners in this respect seem almost superhuman to the casual player. For instance, some glitches require 'frame perfect' or 'pixel perfect' inputs. 'Frame perfect' refers to inputs that must be entered between two frames of the game. Since the frame rate of many of the most popular speedrun games is around $30 \mathrm{fps}$, this means that a frame perfect input must be within a 0.03 second window. A 'pixel perfect' trick is one in which buttons must be pressed when the player's avatar occupies specific pixels. Since even older systems such as the Famicom/Nintendo Entertainment System have 61,440 pixels on screen at any time, this kind of technique involves exceedingly precise control.

The control and precision required by speedrunning is also seen in the way that top players regularly incorporate Tool Assisted Speedrun (TAS) techniques into their runs. A TAS is a run using an emulator that allows the player to 'slow a game down to individual 
frames (what's known as "frame advance"), allowing them to make inputs precisely' (Snyder $2017,21)$. The player then takes each frame in turn, exploring the results of different inputs. As a result of this, TAS speedrunners can chart the most efficient routes through games, even to the point of established a theoretically perfect run. In TAS runs, 'reflex-heavy tricks that are impressive in real-time become trivial when the game is slowed down to a fraction of a second' (Snyder 2017, 23). Though the insights of TAS research are regularly incorporated into competitive RTA running, certain tricks and glitches discovered through TAS are frequently deemed beyond the abilities of any human player to implement in real time. However, runners have often spent months learning how to reproduce supposedly impossible tricks reliably in real time, to the point where they become standard techniques required for a run to be world-record competitive.

The difference between long runs can be measured in minutes: the three fastest times on speedrun.com in the 'All Moons' category of Super Mario Odyssey (2017) are 16 minutes apart in a run that takes over seven and a half hours. For medium length runs, players compete over mere seconds: the top three places on speedrun.com in the Super Mario 64 (1996) ' 120 -star' category differ by only 21 seconds in an approximately $1 \mathrm{~h} 40$ minute run. World record breaking runs in short categories are usually by fractions of a second: the top three times on speedrun.com in the Super Mario Bros (1985) 'Any\%' category, for instance, are all within $150 \mathrm{~ms}$ of each other. Earning a world record score therefore requires nearperfect execution and extremely precise control, sometimes over long periods of time.

Dexterity is not the only skill involved in speedrunning, however. There are also a range of mental skills involved. For instance, the player must have committed their route-their path through the game-to memory. This includes fine details of the game, to the point where the runners know the game far better than the programmers who designed it. For many games, and especially those games with significant RNG, on-the-fly scenario analysis is also an essential skill. Finally, required for speedrunning is mental toughness. Speedrunning is a high-pressure activity, requiring runners to complete extremely difficult and precise tricks, one after another, for the duration of a sometimes hours-long run, without error. As David Synder points out,

In popular games like Super Mario 64 the records are so highly optimized that if runners hope to shave off any time at all they can't tolerate even a minor mistake. A mistake leads to a reset, where runners stop the timer and reboot the game, losing all their progress. Whether they were two minutes into the speedrun or two hours, they will restart from the beginning. Obviously, it takes a strong mental fortitude to continue under such punishing circumstances. The high bar and accordingly high standards that runners set for themselves often leads to burnout-complete exhaustion with the game (Snyder 2017, 12).

As such, Snyder argues that,

The real challenge of speedrunning lies not in mastering reflex-heavy tricks or memorizing multi-hour routes but in overcoming the psychological toll of resetting. Whether in the first thirty seconds or the last minute of a two-hour run, most of a speedrunner's attempts will end in failure. A slip of the thumb on the controller, bad RNG ... will cause the current playthrough to be voided (Snyder 2017, 30).

For instance, Super Mario Bros speedrunner, darbian, estimates that he has only completed the game on less than $2 \%$ of his 14,000 runs. According to darbian, 'Anytime you start a speedrun ... the expected outcome is failure' (Snyder 2017, 30). 
Of course, the degree to which these various skills matter differs game-to-game, genreto-genre and category-to-category. However, all these skills are applicable to all speedrunning to one extent or another.

The most obvious place where we can see the centrality of these skills to speedrunning is in the impermissibility of hardware modification and hacking. The main reason these actions are deemed impermissible seems to be that they '[give] individuals an unfair advantage where speedrunning was more of a test of skill' (Brewer 2017,63). The use of special controllers or the modification of the code, for instance, allows players who are less skilled to achieve a lower time than a player who is more skilled. If speedrunning is importantly constituted by particular skills, then it is clear why this kind of cheating is disallowed.

\section{Knowledge}

Skills are only one component of the overall ethos of speedrunning. While significant, I argue that skill is the least important component. More central to speedrunning is knowledge of the game. Knowledge of the game may seem superficially like the skill of memorisation. However, we can distinguish the two by thinking of memorisation as the ability to hold and apply information about the game while playing, whereas knowledge is about the background understanding of how the game functions, separate from its immediate application.

Importantly, this knowledge is also collective rather than individual. While speedrunners compete with each other in order to gain the fastest time, the speedrunning community is, at least in its contemporary form, a 'supportive environment in which a community of gamers share strategy and learn from one another while seeking to better each other's virtuoso performances and conquer the game in their name' (Newman 2008, 129). As such, 'there is a clear sense in which the products and processes of speedrunning are owned by the community rather than any particular individual' (Newman 2008, 130). By 'knowledge', then, I mean this background, collective set of routes, strategies, and so on, rather than an individual's ability to implement that knowledge in a run.

The importance of distinguishing knowledge as a separate component of the ethos of speedrunning becomes clearer when we consider some of the glitches that speedrunners typically use. For instance, sequence breaking glitches such as wrong warps-where the player takes advantage of glitches to skip sections of the game-are common in speedrunning. One extreme example of this is the Script Stack Underflow (SSU) glitch in Grand Theft Auto: Vice City (2002). SSU, 'involves hijacking the internal "pointer" which dictates which code the game should execute. This allows runners to essentially reprogram the game to skip to the end credits'. Once this glitch was discovered, the 'Any\%' record in Vice City quickly went from around 53 minutes to only 12 minutes. Similarly, The Legend of Zelda: Ocarina of Time contains a 'wrong warp' glitch that more than halved the world record in the Ocarina of Time 'Any\%' category, from 46 down to 21 minutes (Snyder 2017, 25).

If we want to claim that speedrunning is fundamentally about skill, this is difficult to square with the acceptance of glitches such as these. After all, these glitches clearly undermine the display and testing of the very skills that speedrunning is supposed to be about. Hence, skill cannot be the most fundamental component of the ethos of 
speedrunning. Instead, I suggest that where skill and knowledge conflict with one another, knowledge always wins out. In cases such as Vice City and Ocarina of Time, then, the fact that the glitches represent the best collective knowledge of the speedrunning community about how the code functions trumps the fact that it reduces the skill required of the players.

It is worth noting at this point that extreme glitches such as these ones frequently result in the creation of new categories of play that exclude them, such as 'no-SSU' for Vice City and 'no IM/WW' for Ocarina of Time. However, this does not undermine my contention that knowledge is more fundamental than skill. The creation of new, glitchless categories does not negate the achievement of the glitched category. For instance, the 'Any\%' category of Vice City is still active, with a new record being posted on speedrun. com only twenty days prior to this article being written. It is not, therefore, that the worldrecord times of the runners who used the glitch were voided by the creation of a new category; the glitch was not deemed impermissible in general. Rather, it was seen to change the nature of the category to the extent where a new category was required in order to preserve some of the enjoyable aspects of the old run for those interested. We can think of this as in some ways analogous to the invention of the front crawl in swimming. Front crawl is now the default style in the freestyle category of swimming, having replaced breaststroke. When it was invented, rather than banning the front crawl due to its superiority to the breaststroke, instead a new category was created for breaststroke specifically in order to preserve the specific skills of that activity.

There are cases, however, in which a glitch 'kills' a category. But what makes the category 'dead' is not that the glitch voided any runs that used it. Rather, when games become heavily optimised, i.e. the RTA runs approach the time of a TAS run, there becomes no further purpose in playing. As Snyder puts it, 'the timer can only go so low. Eventually a category becomes "dead," when a runner obtains a time so optimal that nobody thinks it could be surpassed' (Snyder 2017, 227).

An analogy to the death of a category is if we were able to model with perfect accuracy the body and precise series of movements that would be required to run the $100 \mathrm{~m}$ dash in the shortest possible time that physics allows. If we had a high degree of confidence that a time could not be beaten-that is was the shortest time permitted by the laws of physics - then if someone were to achieve that time, that would be the end of competing for the world record in the $100 \mathrm{~m}$ dash, since no future runner could do better than match the world record time. This is what occurs when a speedrunning categories 'dies': the shortest time possible has been achieved, so there is no further purpose in trying to beat it. Hence, even when categories 'die' because of glitches, this does not indicate that skill is more important than knowledge; merely that that category has been played out.

\section{Subversion}

Again, however, knowledge cannot be the end of the story. We can see why when we examine the fourth category of impermissible action in speedrunning: cheat codes. If code is law, and knowledge is more important than skill, then why should cheat codes be excluded from speedrunning? After all, they express a knowledge of the game, are internal to the code of the game-they do not involve hacking or changing the code in 
any way-and they are, in many cases, the fastest way of completing the game. As such, is it even possible to distinguish between cheat codes and glitches?

One possibility is to argue that cheat codes are in some sense outside the game, whereas glitches are inside the game. But what can this mean? To take level select code in Sonic the Hedgehog (1991) as an example, the cheat code is entered before the speedrun begins. Since speedruns usually start when the player takes control of the avatar at the start of the game, and since the cheat code is entered prior to this point, in a sense this is changing the code of the game, because the code that runs in the first moments of an ordinary game is different from the code that runs if one were to start the game using the cheat.

However, this response has two problems. First, many cheat codes are not activated prior to the game. For instance, in Grand Theft Auto IV (2008) the player can enter secret numbers in their in-game mobile phone to instantly replenish their health, armour and ammunition. Yet on speedrun.com this technique is expressly forbidden in all categories of GTA IV speedrun, glitched and glitchless alike. Second, soft resetting is frequently permissible in speedruns. For instance, 'a save and reset in Ocarina of Time will return Link to his house in Kokiri Forest, saving runners the time of backtracking there' (Snyder 2017, 19). This is known in the speedrunning community as 'save warping'. But if save warping is allowed, why is it also not permitted to start a Sonic the Hedgehog run ordinarily, immediately soft reset the console, and then skip to the final stage using the cheat code? The distinction between these two activities is unclear.

We might say, then, that the impermissibility of cheat codes in speedrunning is an indication that my earlier argument-that knowledge trumps skill-is incorrect. After all, cheat codes do reduce the skill required by the player to complete the run. Perhaps they are excluded for this reason.

However, this argument has problems as well. After all, in glitched speedruns activities that are functionally equivalent to cheat codes - that is, activities that have the same impact on the skill required to complete the game-are permitted. For instance, while the level select code is impermissible in Sonic the Hedgehog, glitches that allow the avatar to skip from near the start of some levels to the end of those levels is permissible. Yet like the level select cheat code, this glitch expressly allows the player to avoid playing the level itself, and therefore undermines speedrunning as a test of skill.

Similarly, cheat codes to recover health, ammunition and armour and not permitted in GTA IV, yet the Infinite Sword glitch in Ocarina of Time-a glitch that makes the avatar effectively invincible-is perfectly acceptable (Snyder 2017, 23). In terms of the effect on the skill required to complete the game, it is difficult to tell the difference between these two things.

Instead, then, I argue that the distinction between glitches and cheats is that the former subverts the game, whereas the latter does not. To 'subvert' the game is a matter of playing the game in a way contrary to-or with no regard for-the designers' intentions. As Scully-Blaker puts it, '[g]ame spaces are intelligently designed, strategic spaces that make operational assumptions about how players could or indeed should interact with them' (Scully-Blaker 2016, 47). Speedrunning revels in subverting and disregarding those assumptions. This subversion is, I argue, the central and most fundamental component of the ethos of speedrunning. ${ }^{6}$ 
Subversion is much more obvious in glitched runs. However, there is no speedrunning that is not in some sense subversive and deconstructive. For instance, as a casual player of both Super Mario 64 and Super Mario Odyssey, watching speedrunners skip over carefully constructed puzzles and challenges in glitchless play using nothing more than the basic jumping mechanics of the games can be quite shocking.

Another example is the 'No Major Glitches' category of The Elder Scrolls III: Morrowind (2002). Morrowind is a role-playing game in which players explore the fictional island of Vvardenfell. The sheer size of the game is remarkable: the traversable map is estimated at 9.3 virtual square miles, and the game contains hundreds of Non-Player Characters (NPCs) to interact with as well as nearly four hundred and fifty quests to complete. One important aspect of this game is the exploration of the fictional island's elaborate history, politics and culture. Given the amount of time that the designers must have spent creating these things, doing this is clearly an intended-and central-pleasure of the game. At the time of writing, the world record in the 'No Major Glitches' category on speedrun.com is 4 minutes and 56 seconds. There is no doubt, I would argue, that this subverts the intentions of the creators.

In other words, even glitchless runs are not truly glitchless. 'Glitchless' should instead be taken to mean 'selectively excluding certain glitches', usually with the intention of preserving the enjoyment of the runners. To my knowledge there are no categories in speedrunning that are glitchless in the sense of excluding all-or even most-glitches. It would be a mistaken, therefore, to think that the exclusion of certain glitches is some categories undermines the subversive ethos of speedrunning. Whether glitched or glitchless, the programmer's intentions do not matter: the only reason to exclude certain glitches in glitchless categories is to prevent the exclusion from the game route of particular challenges in the game that the players find enjoyable. Aside from that consideration, however, the players subvert the programmer's intentions however they can.

We might say, then, that speedrunners have taken Roland Barthes' 'death of the author' to heart (Barthes 1977). Speedrunning is fundamentally about disregarding and actively subverting the author's intention. In fact, Scully-Blaker argues that subversion-or 'deconstruction'-is so central to speedrunning that it would simply not be possible to design a game for speedrunners that already contained glitches (Scully-Blaker 2018, 85). As he puts it,

even if, one day, someone made a game that deliberately has glitches left in for speedrunners to use, runners would still undoubtedly play it in a manner that differs from the developerintended path (Scully-Blaker 2018, 85).

In this sense we can think of speedrunning as an activity that, at its most fundamental level, is about taking an environment that has been created-and that has been created with a certain idea of how it should be interacted with in mind-and entirely ignoring those intentions in order to create a new activity of the player's own making. In this sense, then, ignoring the creator's intentions and subverting them amount to more or less the same thing, in the same way that using a window instead of a door to enter and exit a house subverts the intentions of those who made it. The central value of speedrunning is to take what has been provided and push it to its limits. If the game environment is not being pushed, it is not speedrunning. Hence, as Scully-Blaker discusses, even a game that included glitches for speedrunners to find would nevertheless itself being pushed and 
engaged with in a way that the programmers did not intend. Like parkour, interacting with an environment in the way that it was intended to be interacted with by the creators defeats the purpose of the activity (Ford 2018). A space created intentionally to be used as a parkour course, regardless of the skill required to traverse it, would simply not be in the spirit of parkour. Similarly, regardless of how skilled the player may need to be to discover and implement intended 'glitches', ${ }^{7}$ the fact that the player is merely interacting with the game environment in the way the programmers intended undermines it as a true expression of speedrunning, since the player is following a script rather than finding their own path through the space.

Hence, if collective knowledge of the game trumps skill, we can see that subverting the intentions of the programmers trumps knowledge in turn. Cheat codes are not excluded because they undermine the constitutive skills of the game; they are excluded because they undermine the constitutive skills while being intended to do so by the programmers. Glitches, by contrast, even when they undermine the constitutive skills of the game, do so in a way that the programmers did not intend. Hence, I argue that subversion is the fundamental value that structures how we ought to understand the rules and constitutive skills that make up speedrunning.

\section{Speedrunning and Sport}

In this section of the paper, I consider speedrunning in the context of the discussion around whether video games (such as e-sports) can ever be thought of as sport. However, my intention is not to offer and argue for a particular view as to whether speedrunning is a sport. Instead, my interest in considering the issue is to illustrate some of the distinctive features of speedrunning as a gaming activity discussed above; specifically, the importance of collective knowledge and subversion to speedrunning.

My lack of interest in whether speedrunning is a sport is not because the status of e-sports and speedrunning as sports does not matter. As Johansen and Thiborg put it, 'being a sport makes [an] activity automatically legitimized and accepted' (Jonasson and Thiborg 2010, 293). In practice a great deal can ride on whether e-sports in general, and speedrunning in particular, is understood to be a sport, or whether they are 'merely' games. If speedrunning is a sport, then speedrunners' activities are legitimised; they are not playing a 'childish' game but engaging in a valid pastime acceptable for adults. There are also clear implications in terms of funding and sponsoring to consider.

In other words, socially and institutionally it matters whether speedrunning, or e-sports more generally, are considered sports. However, this is not the focus of this paper. To paraphrase Jim Parry, whether or not speedrunning '[is] in some sense "desirable" ... is a separate question' (Parry 2019, 5) from whether or not it is a sport, which is turn a separate question from the issue of what the distinct ethos of speedrunning is.

Nevertheless, considering these questions can shed light on this latter issue. As such, I will here examine some ideas of what constitutes a sport, consider e-sports in light of those ideas, and then discuss these ideas in relation to speedrunning. In doing so, I hope to draw attention to some of the more interesting features of the ethos of speedrunning.

Though it is not universally accepted as a definition of sport, Suits' understanding of sport is nevertheless a useful place to begin. According to Suits, a sport must involve (a) the presence of skill; (b) physicality; (c) a wide following; and (d) stability (Suits 2007, 5). 
Here I focus my attention of the first two criteria, as they help to shed the most light on the ethos of speedrunning. The popularity of speedrunning and its institutional status, while interesting, will be put aside for this discussion. In this section I also consider the virtuality of both speedrunning and e-sports more generally and what this can tell us about sport, e-sport and the ethos of speedrunning.

\section{Skill}

According to Suits' understanding of sport, 'all sports appear to be games of skill rather than games of chance' (Suits 1995, 12). As such, if speedrunning and/or e-sports are fundamentally a matter of chance rather than of skill, then this would disqualify them both from being sport.

As it happens, randomness is an element in (most) e-sport and speedrunning. There are almost always aspects of the games that operate according to RNG. For instance, in the e-sport Starcraft, where your SCV (basic construction unit) exits a building after construction is determined randomly. On occasion, this can mean that your SCV exits the building in a 'trapped' location, i.e. in an area with no exit, effectively making the unit useless. It is possible for this to significantly affect the outcome of games. In Final Fantasy VII speedrunning (and other similar speedrun games), the difference between a world recordpace split and a huge setback can be the number of encounters faced while traversing a section of the map, or which attacks a boss happens to use, both of which are determined randomly.

Of course, some games have much more RNG than others, and I think it fair to say that RNG is probably less of an overall issue in e-sports than in speedrunning. Depending on the game, RNG can be decisive in speedrunning. For instance, in the Final Fantasy VII case, 'good' RNG is a necessary (though not sufficient) condition of making a world record run.

In short, we might say the following: sports are games of skill, not luck. While luck can be a factor in e-sports, generally speaking (though not always) it is a minimal one. However, in speedrunning, depending on the game, RNG can be quite significant. Hence, though we cannot describe speedrunning in general as a game of chance, in some cases it may seem like this description is apt. Presumably, such games should not be considered sport for this reason.

In my view, however, the apparent disanalogy between sports and speedrunning in this case is misleading. While the idea that games of chance are not sports is plausible, it would be a mistake to think that chance has no place in sport. The issue is, rather, whether the game is predominantly, or perhaps fundamentally, a matter of skill or a matter of chance.

For instance, the bounce of the ball in rugby, or how the cricket ball bounces off a worn pitch is, in many cases, effectively random. Yet receiving a favourable bounce can be the difference between winning and losing a match. Nevertheless, we would surely not claim that rugby or cricket are game of chances (and therefore not sport). Of course, in rugby and cricket the ball is not truly random; the bounce is determined by the laws of physics and can therefore, in principle, be predicted. In fact, controlling the way the ball bounces can often be an essential skill. However, to pretend that it is physically possible for the players to control the bounce of the ball in all circumstances is unrealistic. 
For example, though it may be possible for skilled rugby players to control the bounce of the ball in the case of a short chip kick, it is not credible for the players to claim that they have controlled the bounce of the ball when they have made a long kick down the field. However, it is not uncommon for tries to be scored after receiving a favourable bounce from such kicks. If so, then randomness does play a role in rugby-and sometimes an important role. Yet this does not disqualify it from being a sport.

Similarly, random encounters in Final Fantasy VII are not truly random: they are generated by a range of factors interacting in the background that may appear random to the player but are not truly so. In fact, taking advantage of the fact that what is apparently random in such games is in fact not is a big part of the game mastery of the speedrunner. For instance, knowledgeable players of Final Fantasy VII know that, in certain parts of the game, opening and closing the menu in specific locations alters particular background variables, thereby allowing them to avoid 'random' encounters in areas where the odds are very much against doing so.

The point of mentioning this is not, then, to suggest that randomness plays no role in speedrunning. It certainly does (as it does for many sports as well). Rather, it is intended to draw attention, again, to the fact that shared knowledge is a key part of the ethos of speedrunning, and that skill is subordinate to it. In rugby, for instance, the ability to control the bounce of the ball on chip kicks-or to predict its bounce on long ones-is part of the skills of the game. It is not a matter of shared, universally accessible background knowledge, but of ability. In speedrunning, by contrast, manipulating the supposedly random elements of the game is not a matter of skill, but rather a matter of having a deep knowledge of the game mechanics-of the code. This knowledge can be separated from skill-based activity, since it is, in a certain sense, passive. ${ }^{8}$ In other words, speedrunning differs from sports in that passive, shared knowledge of the game's physics, including the way that the game pseudo-randomly generates RNG, matters in speedrunning in a way it does not in sport.

\section{Form of Competition}

Somewhat relatedly, we might suggest that speedrunning is not a sport in that, even though it involves skill, it does not involve competition of the 'right' kind to test those skills. In sport, competition is usually direct. That is, the players or teams are in immediate competition with the other players or teams. This is why, as Mariona Rosell Llorens points out, not all video games should be considered e-sports (Llorens 2017, 467). The video games that are considered under the banner 'e-sport', and that are most plausibly sports, are multiplayer games which, like sports, involve direct competition. By contrast, other video games do not have an opponent (aside from the $\mathrm{Al}$ ), and are more in the nature of 'shadowboxing' than true sport. In Llorens' view,

not all video games engage in e-sport gaming sports practice. World of Warcraft or Diablo (by Blizzard Games) for instance, are highly popular competitive and online video games. However, they are not fundamentally constituted as competitive personal interaction, but rather as a 'profile upgrading' exercise. That is to say that, within such games, there is no final victory, merely an ongoing state of contesting with various opponents. Therefore, it may be argued that these games never have an ending and thus the result-oriented competition requirement for sport is not met (Llorens 2017, 467). 
Of course, as Llorens mentions, it is possible to compete in non-multiplayer games, and speedrunning involves just such competition through comparing single-player achievements. Llorens does therefore view such competition as a forerunner of e-sports. But a forerunner is not the thing itself. Hence, in Llorens' view, speedrunning should not be considered a sport.

In response to this apparent disanalogy between speedrunning and sport we might point to sports such as bobsledding. Bobsledders do not compete directly, if by 'directly' we mean 'simultaneously'. Instead, each team takes turns on the same track and the times of each team are then compared. The same seems to be true of speedrunning: even if players do not compete simultaneously, they nevertheless compete directly in the same way bobsledders do.

However, this response overlooks and important difference between speedrunning and sports such as bobsledding: in bobsledding the number of attempts each team has available to them is explicitly specified and limited. In bobsledding, for instance, each team is given four runs, with the winner being determined by the fastest total time. In speedrunning, by contrast, the runner can make as many attempts as they have the time and patience for, and only the best time 'counts'. In this regard, then, while multiplayer e-sports might be considered a sport, since they aim 'to beat the opponent', with the competition 'settled in discrete matches (i.e. defined time and play sets)' (Llorens 2017, 467), the same is not true of speedrunning. Hence, in this respect speedrunning is perhaps not a sport nor an e-sport.

Nonetheless, identifying this difference sheds some important light on the nature of the speedrunning ethos. For one thing, it foregrounds the value of perseverance and mental toughness. A speedrunner, to win a world record, must make attempt after attempt, with no guarantee that victory is even possible for anyone. In direct competition, there is usually always a winner. ${ }^{9}$ In speedrunning, however, the top time may never be beaten, despite considerable effort by other runners. The knowledge that one's efforts might end up being hopeless is therefore an important part of speedrunning and is something that top runners have to overcome if they are to have any hope of beating the world record time.

Secondly, this difference between speedrunning and sport/e-sport draws attention again to the importance of shared knowledge-and, importantly, the fact that this knowledge is collaborative rather than competitive. If game dexterity and skill were the central value in speedrunning, discrete matches would make more sense. Discrete matches, after all, enable the players to demonstrate their ability to 'perform' the game skills and to do so in direct competition with other players. However, discrete matches are not a central part of speedrunning. Hence, we can see the importance of the shared knowledge of the game to speedrunning, including the runners' 'strats' (strategies/game paths) that rely on knowledge shared with, and often created in collaboration with, the speedrunning community. A top speedrunner is not simply the player who is the most skilled. While they must be skilled to be a top speedrunner, equally important is their ability to implement collectively designed strats that display the deepest knowledge of the game. This knowledge is developed by the community with the shared goal of 'jointly push[ing the game] to the limit' (Snyder 2017, 76). Hence, we should think of shared knowledge (as well as the subversion of the programmer's intentions for the game this displays) as a key value in speedrunning. 


\section{Sport as Physical Activity}

Perhaps the most discussed criterion of sport in the context of e-sport is the (supposed) requirement that sport involve physical activity. Certainly, the requirement that sports have a physical component is emphasised by many sports philosophers, including Suits.

In his discussion of whether 'mind sports' such as chess are sports, Filip Kobiela identifies four possible positions that one could take in relation to this claim (Kobiela 2018). The same typology can be easily applied to the question of whether e-sports and/ or speedrunning are sport. First, the exclusivist position holds that utilising physical skill is a requirement for e-sports and/or speedrunning to be a sport, but that e-sports and speedrunning do not, in fact, rely on physical skills (or that those skills are physical in the right way). Second, the conservative inclusivist agrees with the exclusivist that e-sports and/or speedrunning can only be considered sports if they are physical in the right way, but contra the exclusivist argues that they are physical in the right way. Third, the radical inclusivist accepts that e-sport and/or speedrunning are not physical in the 'right' way but denies that utilising physical skill is a necessary criterion of sport. Finally, the double inclusivist holds both that e-sport and/or speedrunning is physical and that physicality is not a necessary criterion of sport.

At least some of the discussion about e-sports is between exclusivists and conservative inclusivists. One dispute is therefore whether e-sports are physical in the right way. Physical in the 'right way' is typically understood as meaning that physical skill is integral to the activity, in the sense that it influences the outcome. In the discussion of mind sports, for instance, Suits points out that 'it is a plain fact that how chess pieces are moved has nothing whatever to do with manual dexterity or any other bodily skill' (Suits 1995, 12). In other words, in chess, while the moving of the pieces is physical, how that movement is accomplished does not determine the outcome of the game. As Klaus V. Meier points out, in games like chess or bridge,

Assistants or even machines can move the pieces or display the cards; verbal instructions or commands may suffice and, in fact, chess can be played by mail (Meier 1995, 25).

By contrast, in sport the physical movement of the player is integral to successful action. For instance, as Seth E. Jenny et al. put it,

When playing basketball, the manner by which one performs a shot will have a direct impact on whether the shot is successful. A poorly executed jump shot will likely end with a resultant miss (Jenny et al. 2016, 9).

For some, such as Dennis Hemphill, e-sports (or, as he refers to them, 'cybersports') obviously do involve physical skills. He notes that 'there are certain bodily movements, including various motor skills, that must become in a sense "automatic" in order for an athlete to execute tactics and strategies' (Hemphill 2005, 203). If this is the case, then clearly e-sports have a physical component in the right sort of way.

However, this is not generally taken to the end of the story. Even if relationship between the physical action and the outcome is of the right kind, there is the question of whether the physical action itself is of the right kind. This disagreement is generally framed in terms of 'fine' vs. 'gross' motor skills. The argument by exclusivists is therefore that by 'physical skills' we ought to mean 'gross motor skills' rather than 'fine motor skills'. 
According to Hemphill and Klaus V. Meier, one central problem with defining physical skill exclusive of fine motor skill is that the distinction is difficult, they think, to uphold in practice. Hemphill asserts, for instance, that 'the mind boggles when considering where you draw the line between a gross and fine motor skill' (Hemphill 2005, 199), and Meier adds that '[a] distinction between fine and gross motor activities, as a criterion for distinguishing sports from games, is ... arbitrary, unnecessary, and counter-productive' (Meier 1981, 85).

By contrast, exclusivists such as Jason Holt and Seth E. Jenny, et al., argue that distinguishing between gross and fine motor skills is actually rather straightforward. They suggest that gross motor skills, 'include skills in which large muscle groups (e.g. quadriceps, hamstrings, gluteus maximus, etc.) produce movement', such as 'kicking a soccer ball, performing a gymnastics routine, or jumping over a hurdle'. Conversely, fine motor skills 'involve precise movements with increased accuracy and control which utilize smaller muscle groups', and that 'skills in which one manipulates an object (e.g. video game controller) involves fine motor skills' (Jenny et al. 2016, 9). Hence, e-sport is not a sport, since it does not involve (clearly defined) gross motor skills: for Holt, 'it is not the right sort of physical game' (Holt 2016, 7). There is no debate, therefore, about whether e-sport is skilful. The question is, then, whether it is skilful in the right way.

But how does this discussion apply to speedrunning? There is one important difference between e-sports and speedrunning: as has already been mentioned, e-sports is a discrete performance, whereas speedrunning is an ongoing, unbounded effort. When it comes to skills, including fine motor skills, while they are essential to both e-sports and speedrunning, we might say they are necessary and sufficient in e-sports, but merely necessary in speedrunning.

If we include skills such as team work, the ability to react rapidly, and so on, along with fine motor skills in our understanding of 'skill' in video games, we can see that the constitutive skills of e-sports are what e-sports fundamentally tests. This is not true in speedrunning, where skill that is not paired with a deep knowledge of the mechanics of the game is not sufficient. To put things another way, we might say that in e-sports, the game exists merely as a way of testing certain constitutive skills. In speedrunning, the game does test certain skills, but also exists as an object of knowledge itself, and that deep knowledge of the game in and of itself is a part of what speedrunning is about.

\section{Virtual Sport}

Despite the distinction between fine and gross motor skills, there are some tricky borderline cases when it comes to e-sport. For instance, there are systems like the Wii, Kinect and Move, all of which require gross physical movement as a way of interfacing between the player and the game. Would an e-sport tournament using these kinds of interfaces count as a sport? Holt, for one, suggests that 'it is hard to see on what principled grounds we could exclude such games from the sport class' (Holt 2016, 8).

One way even videos games that utilise gross motor skills might be excluded from the category 'sport', however, is by drawing attention to the distinction between the domain of execution and domain of application when it comes to the display of these gross motor skills (Holt 2016). According to Holt, we can distinguish sport from e-sport by noting that in sport, the domain of execution-where we perform the action-and the domain of 
application-where the outcome takes place-are both actual. By contrast, in e-sport the domain of execution is actual, but the domain of application is virtual. According to Holt,

In ordinary sport, the domains coincide on the actual playing field: where I take the shot is also, unremarkably, the same realm in which I aim to score a goal. But this cannot be the case for cybersport, which by definition requires that skills be executed in one domain, which is actual, and then transposed into a virtual one (Holt 2016, 7).

Perhaps, then, the distinction between domain of application and execution is a 'theoretical basis for the intuition that it is the virtuality of the videogame environment, in contrast to the physicality of the traditional sport environment, that prevents inclusion of videogames in the sport category' (Holt 2016, 7).

In respect to this issue speedrunning is in the same boat as e-sport. However, considering speedrunning in light of the distinction between domain of execution and domain of application allows us to draw attention to some important features of speedrunning. For one thing, it reinforces the idea that the games themselves should be thought of as a set of physics rather than as a collection of rules. As Llorens notes, 'the " $\mathrm{e}$ " of electronic is ... the medium through which the sport is played, akin to water being the medium of water polo, or astroturf the medium of modern field hockey' (Llorens 2017, 470). By thinking of the domain of application as just that-a domain, albeit a virtual one -we can see that we ought to treat the game the same as 'any specific medium that a sport may have' (Llorens 2017, 470), such as the water of water polo.

In response to this, a critic may argue that, if so, 'videogames lack constitutive rules so characterized' (Holt 2016, 8). For instance, in Holt's words, 'where we refrain from breaking breakable rules in a footrace or chess game, there do not appear to be such essentially breakable rules that define videogame play', since 'the programming that defines what may legally happen in the virtual domain of a videogame is precisely also what defines that very environment' (Holt 2016, 8). As such, if we think of the code itself as physics, then if Holt's argument is right then speedrunning is potentially not even a game. After all, 'if one cannot break the rules, then they are not game-constitutive rules-they are regularities more like the laws of nature than regulations per se' (Holt 2016, 8). Of course, that the code is like laws of nature-like physics, even if only figuratively-is precisely what I have been claiming. Since anything goes in glitched speedrunning, so long as it is consistent with the code/the virtual physics of the game, then Holt's argument raises serious questions about speedrunning's status as a game in the first place.

However, the distinction between the domain of execution and domain of application helps us to clarify precisely where this criticism goes wrong. There is an important disanalogy between speedrunning (and e-sports more generally) and sport: in sport, the domain of execution and application are identical; in video games they are not. It is precisely because the domain of execution and application are the same in sport that constitutive rules are required to limit what actions the player can perform. In video games, however, the player interfaces with a virtual world: the domain of execution and application are separate. Because of this, the interface itself acts as the constitutive rules that limit the player's action. This is reflected in the fact that many of the most significant rules in speedrunning are around how one can interface with the virtual world; that is, which controllers are permissible, the unacceptability of code manipulation, and so on. These limitations might be thought to take the place of the constitutive rules of sport. 
In other words, the code of the game should be thought of as the physics, rather than the rules, of the virtual worlds that players engage with in speedrunning. But because player actions do not directly correspond to outcomes in that virtual worldpressing a button in the actual world leads to, for instance, the avatar swinging a sword in the virtual one, rather than a one-to-one reflection of the player's physical movement -then the restrictions around how the player can interface with the virtual world matter in much the same way as the constitutive rules that restrict our actions in sport. At the same time, this distinction shows the limitations of the physics metaphor, since, unlike in sport, the 'physics' of the game is not all there is to e-sport or speedrunning: unlike in sport, the physics of video games must be interfaced with by a player who exists in a world with an entirely different set of physics from the game. In other words, it is the game physics, the players actions in the real world, and the nature of the interface in combination that is important in speedrunning, and that constitutive a complete description of the activity.

The distinction between the domain of execution and application also helps us to draw attention to a mistaken way of considering rules in e-sports and speedrunning: programmers are not rule-makers; they are not gamewrights in a philosophical sense, yet are often confused as such. For instance, Cem Abanazir writes that 'in the case of e-sports, the video game too consists of rules, i.e. the code, allowing certain moves but limiting others', and that 'cases sanctioned and prohibited moves of a player are provided for by the rulemaker, [the programmer], which has the power to produce and/or alter the rules of the game' (Abanazir 2019, 121). In other words, for Abanazir, 'the code-writer, i.e. the developer, is the rule-maker' (Abanazir 2019, 122).

Even if this claim is true in the case of e-sports (and I would suggest that it is not), it is certainly not true of speedrunning. This is precisely what makes speedrunning a metagame rather than a game. The game is simply the medium through which the metagame is played; it is a tool, nothing more. Instead of gamewrights, programmers and developers should be thought of as equivalent to the people who design racetracks for athletics or motorsports, build the swimming pools for aquatic sports, or build and maintain the pitch in sports like cricket. This analogy is not a perfect one, since programmers and developers have more freedom to build as they like than, say, the builders of swimming pools (since the pools have to meet certain standards determined by the real gamewrights). Nevertheless, the point remains that in speedrunning the game is an environment with which the player interacts, not a set of rules. This is precisely why, in glitched speedrunning at least, the programmer's intentions do not constrain what is considered acceptable by the speedrunning community. The programmers simply have no authority to determine what is and is not allowed once the game is out in the world; their only role is in making the game into a certain kind of tool/environment.

It also explains why speedrunners, unlike e-sports players, often ignore the programmers when they try to remove glitches or change the game according to their (the programmer's) preferences. If the speedrunning community does not think that the updates and changes improve their experience of the game, or that they render newer and older times incomparable, the community will simply disallow those changes and require that all runners utilise an older version of the game. If the programmers were truly the gamewrights, this would not make much sense, since it would mean that the speedrunning community are no longer 'truly' playing the game. 
In this regard, again, speedrunning can be usefully compared to parkour (Ford 2018). It involves taking an environment that someone else has provided and using in in ways contrary to the intentions of the creator. In parkour this involves climbing or otherwise bypassing structures intended to be circumlocuted or interacted with in specific ways; in speedrunning, it means using glitches that the programmer was likely unaware of to forge the player's (or, really, the speedrunning community's) own path through the game. In other words, essential to speedrunning is the idea that the runners are not limited by the intentions the creators had for their virtual world; that, in fact, the virtual world should be pushed past its limits in a way that actively subverts the intentions of the programmers. Speedrunning is precisely the act of 'sidestepping the intended and expected rules of playing the game as defined by the designer in order to redefine the goals of the game for their own purposes as a community' (Cook and Duncan 2016, 185). For this reason, the value of 'subversion' has an essential place at the heart of the speedrunning ethos.

\section{Conclusion}

In summary, speedrunning is a unique kind of metagame that utilises the game as the background 'physics' against which the metagame is played. This background physics helps to constitute the metagame (as the background physics of sport is a-usually unacknowledged-part of the constitution of physical sports). In speedrunning, any possible action according to that physics is also a permissible action (at least in glitched categories of play).

Despite this, the player is limited in how they interact with that physics by rules around hardware use. They must also refrain from altering that background physics - the code-in any way (a practical impossibility in physical sports, but not in speedrunning). This is because speedrunning is in large part constituted as a test of skill: in particular, dexterity and finegrained manipulation of the game via the controller; memorisation; and mental fortitude. These skills are a central part of what makes speedrunning the metagame that it is. They therefore provide a central part of the background conventions and purpose of speedrunning that stand behind rule making and rule interpretation in the speedrunning metagame.

However, more important than these constitutive skills is collective knowledge of the game. If a deeper knowledge of the game brings to light glitches that undermine the constitutive skills of speedrunning, then (in glitched categories at the very least) those glitches are permissible. Even in glitchless categories (more properly understood as 'no major glitches', since many glitches are still allowed in most so-called 'glitchless' categories), the display and testing of the constitutive skills of speedrunning is frequently undermined. Hence, collective knowledge can be seen to be more central to the ethos of speedrunning than constitutive skills. It influences how constitutive skills end up managing and applying rules in speedrunning.

Finally, however, the role of collective knowledge in the ethos of speedrunning is limited by the more fundamental goal of subverting the intentions of the game designers. As we can see in the blanket impermissibility of cheat codes in speedrunning, if knowledge of the game undermines the constitutive skills of speedrunning, the question then becomes whether it does so in a way that subverts the programmers' intentions and interacts with the game environment according to the player's (or community's) own reconfiguration of that space. If it does not, then that action is impermissible. If it does, however, then the situation 'reverts' to 
one in which skill is the main consideration (since knowledge has been excluded as a factor). In this sense, then, the desire to subvert the intentions of the programmers in speedrunning play is the most basic feature of the general ethos of speedrunning. While both are extremely important parts of the speedrunning ethos, constitutive skills and collective knowledge are limited and structured by this goal of subversion.

\section{Notes}

1. Of course, the code 'allows' one to hack and modify it, in the sense that it does not resist it; it is something that can be done. Here, therefore, I limit "allows" to those actions that involve ways that the code can be implemented without changing the code itself. By analogy, a book allows us to read the chapters it contains in any order; it also 'allows' us to rip out pages, or to insert our own pages with glue. But there is a clear difference between interacting with the content of the book in a radical way and physically altering the book itself.

2. As I discuss later in the paper, calling the game code 'physics' is, of course, figurative. However, I think it is a useful analogy nonetheless.

3. Again, while there were human-made intentions that the wall be interacted with in a particular way, these do not have the status of rules.

4. It may be pointed out that the Fosbury Flop was consistent with the intentions of the highjumping gamewrights, whereas the use of glitches presumably is not consistent with the intentions of the programmers. However, it should be noted again that speedrunning is a metagame. The game that speedrunners are playing is not the game that the creators intended, and the programmers should not be considered the gamewrights of speedrunning. Instead, the speedrunning community itself are the gamewrights; they create the rules surrounding how the environment provided by the programmers can be interacted with, and it is their judgement as to an action's acceptability that matters, not the programmers'. If we reject the idea that the programmers are the gamewrights, then the two cases are no longer disanalogous in this way. The programmers make the game, but they do not determine how it is used. Instead, that is a matter for the speedrunning community to decide.

5. And I think it would be fair to say that, just like changes to code in speedrunning, if a swimmer swum in a liquid other than water, or in a pool on the moon, even if they broke the world record while doing so it is unlikely that the times from such activities would be considered directly comparable to 'ordinary' swimming.

6. The sense of 'subversion' I am using here excluded hacking. Subversion in the speedrunning ethos is about taking what is given and reconfiguring it. To use the book analogy again, subversion is to read the words in a different order; hacking is changing the words.

7. Though arguably an intended glitch is more a 'cheat' than a true glitch.

8. By 'passive' here I mean knowledge that can be applied as described. For instance, being told how to predict the bounce of a ball in rugby is not enough to actually be able to do so in practice: it requires skill. By contrast, being told how to manipulate the random elements in games is frequently enough to be able to do so successfully. Of course, in practice things are not so simple, since some RNG manipulation in speedrunning is technically quite difficult. Nevertheless, the distinction is, I think, a useful one.

9. Draws in cricket test matches notwithstanding.

\section{Acknowledgments}

I would like to thank Don Oxtoby for his helpful comments on an early draft, Bevan Marten for suggesting the Fosbury Flop example, and the reviewers for their extremely useful feedback. Most of all, I would like to thank Aki for watching many moons-worth of Mario with me as "research" for this paper. 


\section{Disclosure Statement}

No potential conflict of interest was reported by the author(s).

\section{ORCID}

Michael Hemmingsen (D) http://orcid.org/0000-0001-9436-139X

\section{References}

ABAnaziR, c. 2019. Institutionalisation in E-sports. Sport, Ethics and Philosophy 13 (2): 117-31. doi:10.1080/17511321.2018.1453538.

BARTHES, R. 1977. The death of the Author. In Image, music, text. Translated by Stephen Heath. New York, NY: Hill \& Wang: 142-148.

BREWER, C.G. 2017. Born to run: A grounded theory study of cheating in the online speedrunning community. Master's Thesis, University of Southern Mississippi.

COOK, L. and S. DUNCAN. 2016. 'Any\% no sketch glitch': Speedrunning final fantasy VI and expanding 'well played'. Well played: A Journal on Video Games, Values, and Meaning 5 (2): 173-89.

D'AGostino, F. 1981. The ethos of games. Journal of the Philosophy of Sport 8 (1): 7-18. doi:10.1080/ 00948705.1981.9714372.

FORD, D. 2018. Speedrunning: Transgressive play in digital space. Proceedings of Nordic DiGRA 2018. doi:10.13140/RG.2.2.12357.91369.

FRANKLIN, S. 2009. 'We need radical gameplay, not just radical graphics': Towards a contemporary minor practice in computer gaming. Symplokē 17 (1-2): 163-80. doi:10.1353/sym.2009.0013.

GDQSTAT. 2020. Awesome games done quick 2020 stats. Available at https://gdqstat.us/?series=0 (accessed 24 April 2020).

GOOD, O.s. 2019. AGDQ 2019's 10 best speedruns from a record-setting week. Polygon. Available at https://www.polygon.com/2019/1/13/18180935/agdq-2019-best-speedruns-video-on-demandmoney-raised (accessed 24 April 2020).

HEMPHILL, D. 2005. Cybersport. Journal of the Philosophy of Sport 32 (2): 195-207. doi:10.1080/ 00948705.2005 .9714682$.

HOLT, J. 2016. Virtual domains for sports and games. Sport, Ethics and Philosophy 10 (1): 5-13. doi:10.1080/17511321.2016.1163729.

ILLINGSON, A. 2019. Activision Blizzard uses Nielsen numbers to draw advertisers to Overwatch League. L.A. Biz. Available at https://www.bizjournals.com/losangeles/news/2019/09/05/activisionblizzard-uses-nielsen-numbers-to-draw.html (accessed 24 April 2020).

JENNY, S.E., R.D. MANNNG, M.C. KEIPER, and T.W. OLRICH. 2016. Virtual(ly) athletes: Where e-sports fit within the definition of "sport". Quest. doi:10.1080/00336297.2016.1144517.

JONASSON, K. and J. THIBORG. 2010. Electronic sport and its impact on future sport. Sport in Society:

Cultures, Commerce, Media, Politics 13 (2): 287-99. doi:10.1080/17430430903522996,293.

KOBIELA, F. 2018. Should chess and other mind sports be regarded as sports? Journal of the Philosophy of Sport 45 (3): 279-95. doi:10.1080/00948705.2018.1520125.

KREIDER, A.J. 2011. Game-playing without rule-following. Journal of the Philosophy of Sport 38 (1): 5573. doi:10.1080/00948705.2011.9714549.

LLORENS, M.R. 2017. E-sport gaming: The rise of a new sports practice. Sport, Ethics and Philosophy 11 (4): 464-76. doi:10.1080/17511321.2017.1318947.

MEIER, K. 1995. Triad trickery: Playing with sport and games. In Philosophic inquiry in sport, 2nd ed., edited by W.J. Morgan and K.V. Meier. Champaign, IL: Human Kinetics: 16-35.

MEIER, K.V. 1981. On the inadequacies of sociological definitions of sport. International Review for the Sociology of Sport 16 (2): 79-102. doi:10.1177/101269028101600206.

NEWMAN, J. 2008. Playing with videogames. Oxon: Routledge.

PARRY, J. 2019. E-sports are not sports. Sport, Ethics and Philosophy 13 (1): 3-18. doi:10.1080/

17511321.2018.1489419. 
PFLeEGor, A.G. and D. ROESENBERG. 2014. Deception in sport: A new taxonomy of intra-lusory guiles. Journal of the Philosophy of Sport 41 (2): 209-31. doi:10.1080/00948705.2013.785424,217.

SCULLY-BLAKER, R. 2016. Re-curating the accident: Speedrunning as community and practice. Master's Thesis, Concordia University.

SCULLY-BLAKER, R. 2018. The Speedrunning museum of accidents. Kinephanos (Special Issue: Preserving Play) 71-88.

SNYDER, D. 2017. Speedrunning: Interviews with the quickest gamers. Jefferson, NC: McFarland and Company, Inc.

SPEED DEMOS ARCHIVE. 2019. Rules. Available at https://kb.speeddemosarchive.com/Rules (Accessed 15 April 2020).

sulTs, B. 1995. Tricky triad: Games, play and sport. In Philosophic inquiry in sport, 2nd ed., edited by W. J. Morgan and K.V. Meier. Champaign, IL: Human Kinetics: 16-22.

sulTs, B. 2005. The grasshopper: Games, life and Utopia. Toronto: Broadview Press. Available at https://www.speedrun.com (accessed 23 April 2020).

sUITS, B. 2007. The elements of sport. In Ethics in sport, edited by W.J. Morgan. Champaign, IL: Human Kinetics: 9-19. 